\title{
T1 mapping, ECV and ICV before and after aortic valve replacement
}

\author{
Emmanuelle Vermes*, Nicolas Cazeneuve, Olivier Genee, Anne Delhommais, Laurent Brunereau, Daniel Alison, \\ Julien Pucheux \\ From 18th Annual SCMR Scientific Sessions \\ Nice, France. 4-7 February 2015
}

\section{Background}

the aim of the study was to assess T1 mapping using T1 values, extracellular volume (ECV) and intra cellular volume (ICV) with cardiovascular magnetic resonance (CMR) in patients with surgical aortic stenosis before and after surgery.

\section{Methods}

A prospective CMR T1 mapping study of 37 patients with severe AS before and six months after aortic valve replacement (AVR) was conducted. CMR at $1.5 \mathrm{~T}$, including T1 mapping using a modified Look-Locker inversion recovery sequence (before and 15 minutes after the administration of $0.2 \mathrm{mmol} / \mathrm{kg}$ of Gadoteric acid), was carried out. Global T1 values, ECV, ICV have been measured before and six months after surgery.

\section{Results}

After AVR, post contrast T1 values were significantly lower compared to before surgery (443 $\pm 18 \mathrm{~ms}$ versus $460 \pm 19 \mathrm{~ms} ; \mathrm{p}=0,027)$. ECV was significantly higher after surgery $(27,9 \pm 4,7 \%$ versus $25,1 \pm 2,7 \%$; $=$ $0.0001)$. Reciprocally, ICV significantly decreased after surgery $(74,9 \%$ versus $72,1 \% \mathrm{p}=0.0001)$. In parallel, myocardial mass $(89.5 \pm 27 \mathrm{~g} / \mathrm{m} 2$ versus $74.5 \pm 23 \mathrm{~g} / \mathrm{m} 2$ ; $\mathrm{p}<0,001)$, end diastolic volume $(74 \pm 19 \mathrm{ml} / \mathrm{m} 2$ versus $65 \pm 12 \mathrm{ml} / \mathrm{m} 2 ; \mathrm{p}<0,001)$ and septal thickness $(15 \pm 2.5$ $\mathrm{mm}$ versus $14 \pm 2.5 \mathrm{~mm} ; \mathrm{p}<0,001)$ decreased significantly after surgery.

\section{Conclusions}

Post contrast T1 values, ICV, myocardial mass and septal thickness significantly decreased after AVR, suggesting that LV hypertrophy regression is more cellular than interstitial fibrosis regression. Larger studies are needed to confirm these data.

\section{Funding}

No funding.

Published: 3 February 2015

doi:10.1186/1532-429X-17-S1-P342

Cite this article as: Vermes et al:: T1 mapping, ECV and ICV before and after aortic valve replacement. Journal of Cardiovascular Magnetic

Resonance 2015 17(Suppl 1):P342.

Cardiac Imaging, CHU Trousseau, Tours, France

Submit your next manuscript to BioMed Central and take full advantage of:

- Convenient online submission

- Thorough peer review

- No space constraints or color figure charges

- Immediate publication on acceptance

- Inclusion in PubMed, CAS, Scopus and Google Scholar

- Research which is freely available for redistribution
() Biomed Central 